\title{
Protocolos de activación y su efecto en la performance deportiva en nadadores recreativos de Viña del Mar
}

http://dx.doi.org/10.11606/1807-5509201900010021

\author{
Juan Pablo ZAVALA* \\ Rodrigo YANEZ ${ }^{* *}$ \\ Fernando Sandoval TIETZ* \\ José Alvarez OPAZO* \\ Fernando Barraza GÓMEZ*** \\ *Facultad de \\ Educación, \\ Universidad Andrés \\ Bello, Viña del Mar, \\ VS, Chile. \\ ${ }^{* *}$ Facultad \\ de Filosofía y \\ Educación, Pontificia \\ Universidad Católica \\ de Valparaíso, \\ Valparaíso, VS, Chile. \\ ***Universidad Viña \\ del Mar, Viña del Mar, \\ VS, Chile.
}

\section{Resumen}

El objetivo del presente estudio fue determinar los efectos de tres protocolos de activación muscular en la fuerza y tiempo en una prueba de 25 metros de estilo libre con salida baja en un grupo de nadadores de nivel recreativo de Viña del Mar, Chile. Participaron del estudio 31 sujetos, con una edad promedio de $21,86 \pm 2,38$ años. Estos fueron divididos en cuatro grupos de participación en base a un método cruzado aleatorio, los grupos se clasificaron en: grupo control (CON), grupo de ejercicio isométrico (ISO), grupo de ejercicios excéntricos y concéntricos (EX - CON) y grupo de movilidad articular (MA). En todos los protocolos se utilizó una carga de entrenamiento equivalente al peso corporal del sujeto. Las variables evaluadas fueron; tiempo en segundos en 25 metros estilo libre con salida baja y fuerza máxima registrada a través del monitor de fuerza modelo $\mathrm{FMON}-1$ de la marca ART-OFICIO ${ }^{\circledR}$. Los resultados evidenciaron que la activación con isometria (ISO) tuvo respuesta positiva $(p=0,020)$ en la variable fuerza $(N)$. Los protocolos de entrada en calor no produjeron disminución del tiempo en la prueba de 25 metros $(p>0,05)$. Estos resultados llevan a la conclusión que la isometría aumento la fuerza en el agua, pero este aumento en la fuerza no reflejó una disminución del tiempo obtenido en el sprint de natación, los demás protocolos no produjeron variación en el tiempo ni en la fuerza, se sugiere seguir explorando los beneficios de una activación previa a un esfuerzo deportivo, y en particular indagar los beneficios de diferentes protocolos de potenciación post activación como intervención previa a pruebas de sprint en natación.

Palabras clave: Natación; Activación; Rendimiento; Fuerza.

\section{Introducción}

Diversos estudios han evidenciado efectos positivos inducidos por la activación previa a la competencia deportiva ${ }^{1-2}$, los profesionales y entrenadores deben considerar el uso de la potenciación con una activación que no produzca efectos negativos en la rigidez del tendón ${ }^{3}$ para no perjudicar el rendimiento en el deportista, se ha visto en modelos animales que el entrenamiento disminuye la rigidez del tendón y aumenta el metabolismo del colágeno ${ }^{4}$, es por esto que los deportistas tienen tendones con capacidad para soportar grandes cargas como las producidas por la potenciación post activación (desde ahora PAP), en cambio, sujetos que practican deporte de manera recreativa no presentan esta misma adaptación, esto condicionaría la aplicación de protocolos de PAP sin carga en deportistas de nivel recreativo ya que una activación con una carga elevada puede producir lesión o disminución del rendimiento deportivos. El uso de los protocolos de activación muscular en los últimos años se ha popularizado, este interés se 
basa en evidencia científica que manifiesta que la función del músculo puede verse favorecida posterior a la aplicación de una activación muscular previa ${ }^{6}$, lo que produciría un incremento en la respuesta muscular contráctil al realizar ejercicios explosivos luego de producir contracciones musculares máximas ${ }^{7}$. La aplicación de la PAP puede considerarse en la natación, donde la fuerza, que se define como la capacidad del hombre para vencer resistencias externas o contrarrestarlas a costa de esfuerzos musculares ${ }^{8}$, se convierte en una capacidad condicionante esencial para poder vencer las diversas resistencias encontradas en el medio acuático, en donde un aumento de la respuesta contráctil puede significar la obtención de una medalla de oro olímpica o el rompimiento de un record mundial, dichos esfuerzos musculares deben ser trabajados de tal forma que tengan una organización orientada a una meta específica, es allí donde juega un rol fundamental la preparación física, la que se define como la aplicación de un conjunto de ejercicios corporales, generalmente ajenos a los que se utilizan en la práctica del deporte' ${ }^{9}$, es en este contexto donde la potenciación post activación adquiere protagonismo como método de entrada en calor debido a sus efectos positivos en la actividad contráctil del músculo, de esta manera se manifiesta la potenciación como una respuesta muscular contráctil incrementada como resultado de una actividad muscular previa ${ }^{10}$. En el ámbito acuático, es Rushall ${ }^{11}$ quien da un giro en la forma de planificar los entrenamientos de natación, que se basaban sólo en trabajar un volumen considerable de nado por sesión, planteando que la natación se debe trabajar en estricta relación con sus pruebas de competición, dando énfasis a una cantidad exacta de repeticiones y sólo centrándose en un estímulo específico. El ya mencionado estímulo cobra mayor relevancia en una actividad previa cuando el deportista quiere alcanzar un máximo rendimiento específico. Con relación a la metodología utilizada en este estudio, BARBOSA et al. ${ }^{5}$ sugieren no aplicar potenciación post activación con cargas altas en nadadores menos experimentados ya que puede producir una respuesta negativa en el rendimiento deportivo, esta referencia da pie a comenzar con esta investigación, utilizando activaciones sin carga para establecer las posibles mejoras de rendimiento en natación. El objetivo de la presente investigación fue determinar los efectos de diversos protocolos de activación en el tiempo (segundos) y la fuerza (newton) en la prueba de 25 metros estilo libre con salida baja en nadadores de nivel recreativo de Viña del mar, Chile.

\section{Método}

La muestra estuvo compuesta por un grupo de 31 nadadores de nivel recreativo con edad promedio de $21,86 \pm 2,38$ (18 a 23 años) escogidos de manera aleatoria. Todos los participantes eran sujetos físicamente activos, sin patologías y con un estado de salud compatible para realizar natación. Se aplicó un muestreo aleatorio donde todos los sujetos realizaron todas las intervenciones, esto permitió comparar los resultados obtenidos en el control con relación a los resultados obtenidos de las intervenciones en base a diversos protocolos de PAP. La investigación comenzó posterior a la revisión del consentimiento informado y el protocolo de la investigación, el cual fue aprobado previamente por los participantes. El protocolo de investigación fue aprobado por el comité de ética de la Facultad de Educación de la Universidad Andrés Bello.

Las evaluaciones fueron realizadas en piscina de 25 metros, techada y temperada a $26^{\circ}$ Celsius, la temperatura ambiente fue de $21^{\circ}$ Celsius. En la primera etapa, todos los grupos realizaron la evaluación de control en una prueba de natación de 25 metros estilo libre, en las siguientes etapas se aplicaron las intervenciones de los diferentes protocolos de PAP (TABLA 2). Las variables medidas correspondieron a fuerza máxima ejercida en el agua expresada en newton $(\mathrm{N})$ y tiempo de nado en sprint de 25 metros estilo libre expresado en segundos (s). La variable de fuerza $(\mathrm{N})$ fue registrada a través de un monitor cuantificador de fuerza modelo FMON-1 de la empresa ART-OFICIO ${ }^{\circ}$ y la prueba de natación en 25 metros estilo libre fue cronometrada en segundos y centésimas. El nadador en el agua llevó un cinturón que tenía un cable de acero conectado al dispositivo de evaluación de fuerza, este cable entregó la información al monitor, logrando así cuantificar la fuerza ejercida durante la prueba de nado. En lo que respecta a la variable tiempo, se evalúo el tiempo de ejecución de sprint 25 metros estilo libre con salida baja.

A continuación (TABLA 1) se presenta el protocolo de intervención utilizado. 
TABLA 1 - Descripción de los protocolos de potenciación post activación (PAP) utilizados

\begin{tabular}{|c|c|c|c|}
\hline \multicolumn{4}{|c|}{ Grupo movilidad articular (MOV) } \\
\hline Movimientos & $\begin{array}{l}\text { Movimiento articular del tren } \\
\text { superior. }\end{array}$ & $\begin{array}{l}\text { Movimiento articular de tren } \\
\text { inferior }\end{array}$ & $\begin{array}{l}\text { Movimiento articular del } \\
\text { CORE }\end{array}$ \\
\hline Ejercicios & Cruce alternado de brazos. & $\begin{array}{l}\text { Flexión de cadera / Extensi- } \\
\text { ón de rodillas. }\end{array}$ & Flexión de tronco. \\
\hline Intensidad & & Peso corporal $(0 \% \mathrm{RM})$ & \\
\hline $\begin{array}{l}\text { Volumen total } \\
\text { de trabajo }\end{array}$ & \multicolumn{3}{|c|}{70 segundos 10 (s) de ejercicio x 10s de descanso) } \\
\hline \multicolumn{4}{|c|}{ Grupo Isometría (ISO) } \\
\hline Movimientos & $\begin{array}{l}\text { Ejercicio isométrico del tren } \\
\text { superior }\end{array}$ & $\begin{array}{l}\text { Ejercicio isométrico tren } \\
\text { inferior }\end{array}$ & $\begin{array}{l}\text { Ejercicio Isométrico } \\
\text { CORE }\end{array}$ \\
\hline Ejercicios & $\begin{array}{l}\text { Flexo-extensión de codo, con } \\
\text { postura isométrica en } 90^{\circ} \text {. }\end{array}$ & $\begin{array}{l}\text { Sentadilla con postura } \\
\text { isométrica en } 90^{\mathrm{a}} \text {. }\end{array}$ & Plancha isométrica. \\
\hline Intensidad & \multicolumn{3}{|c|}{ Peso corporal $(0 \% \mathrm{RM})$} \\
\hline $\begin{array}{l}\text { Volumen total } \\
\text { de trabajo }\end{array}$ & \multicolumn{3}{|c|}{70 segundos 10 (s) de ejercicio x 10s de descanso) } \\
\hline \multicolumn{4}{|c|}{ Grupo excéntrico concéntrico (EXC-CON) } \\
\hline Movimientos & $\begin{array}{l}\text { Ejercicio excéntrico- } \\
\text { concéntrico tren superior }\end{array}$ & $\begin{array}{l}\text { Ejercicio excéntrico- } \\
\text { concéntrico tren inferior }\end{array}$ & $\begin{array}{l}\text { Ejercicio excéntrico- } \\
\text { concéntrico CORE }\end{array}$ \\
\hline Ejercicios & Flexo-extensión de codo. & Sentadilla & Crunch Abdominal \\
\hline Intensidad & & Peso corporal $(0 \% \mathrm{RM})$ & \\
\hline $\begin{array}{l}\text { Volumen total } \\
\text { de trabajo }\end{array}$ & \multicolumn{3}{|c|}{70 segundos (10s de ejercicio x 10 (s) de descanso) } \\
\hline
\end{tabular}

TABLA 2 - Organización de los protocolos de intervención utilizados considerando sesiones realizadas. Distribución de los grupos de manera alternada en base a los tres protocolos de intervención (MOV, ISO, EXC-CON)

\begin{tabular}{|c|c|c|c|c|}
\hline Grupo & Semana 1 & Semana 2 & Semana 3 & Semana 4 \\
\hline 1 & Control & Intervención A (MOV) & Intervención B (ISO) & $\begin{array}{c}\text { Intervención C } \\
(\mathrm{EXC}-\mathrm{CON})\end{array}$ \\
\hline 2 & Control & Intervención B (ISO) & $\begin{array}{c}\text { Intervención C } \\
\text { (EXC-CON })\end{array}$ & Intervención $\mathrm{A}(\mathrm{MOV})$ \\
\hline 3 & Control & Intervención C (EXC-CON) & Intervención A (MOV) & Intervención B (ISO) \\
\hline
\end{tabular}

MOV: movilidad articular; ISO: isometría; EXC-CON: excéntrico y concéntrico.

\section{Resultados}

Fueron evaluados 31 sujetos de la comuna de Viña del Mar. La muestra perteneció a nadadores recreativos con una edad promedio de 21,86 $\pm 2,38$ años. Se presentan los resultados obtenidos en la evaluación de fuerza (Newton) y rendimiento deportivo (tiempo en segundos) en la prueba de 25 metros estilo libre de natación con salida baja. Para comparar los resultados obtenidos entre los grupos se utilizó la prueba T student para muestras relacionadas considerando distribución normal de la muestra con test de Shapiro-Wilk. El nivel de significancia se estableció con intervalo de confianza del 95\% ( $\mathrm{p}<0,05)$. Para el análisis de los datos se utilizaron los software Excel $^{\circ} 2010$ para Windows ${ }^{\ominus}$ y Graphpad $^{\circledR}$ Prism versión 7.

La TABLA 3 muestra los resultados obtenidos en el tiempo y fuerza en prueba de 25 metros estilo libre de natación en los diferentes protocolos realizados. Se observan diferencias significativas entre el grupo control y el grupo isometría en la variable fuerza $(\mathrm{p}<0,05)$. No se apreciaron diferencias entre los grupos en la variable tiempo en 25 metros libres ( $p>0,05)$. 
*Diferencias significativas entre los grupos considerando valor $p<0,05$.

*: Diferencias significativas entre los grupos considerando valor $p<0,05$.

* Diferencias significativas entre los grupos considerando valor $p<0,05$.

TABLA 3 - Media, desviación estándar y valor p de las variables: 25 metros libres (s) y fuerza $(\mathrm{N})$ en los protocolos realizados; control (CON), isométrico (ISO), excéntrico - concéntrico (EX - CON) y movilidad articular (MA).

\begin{tabular}{lcccc}
\hline \multirow{2}{*}{ Variable } & CON & ISO & EX-CON & MA \\
\cline { 2 - 5 } & Media \pm DS & Media \pm DS & Media \pm DS & Media \pm DS \\
\hline Tiempo en 25 metros libres $(\mathbf{s})$ & $20,37 \pm 2,97$ & $20,38 \pm 2,86$ & $20,57 \pm 3,01$ & $20,42 \pm 2,88$ \\
Fuerza en 25 metros libres $(\mathbf{N})$ & $137,11^{*} \pm 27,31$ & $149,10^{*} \pm 39,65$ & $143,60 \pm 36,41$ & $137,13 \pm 31,87$ \\
\hline
\end{tabular}

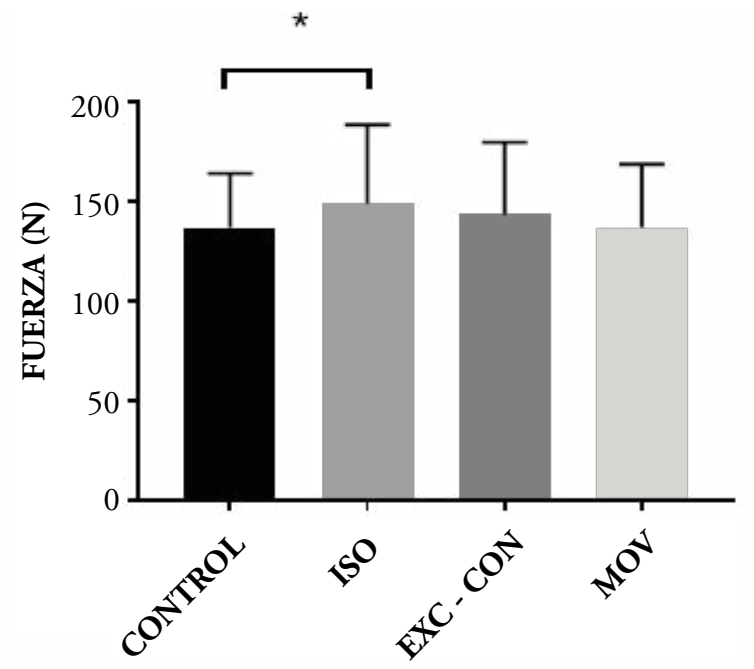

FIGURA 1 - Prueba de fuerza en Natación de 25 metros libres en grupos: control (CON), isometría (ISO), excéntrico - concéntrico (EX - CON) y movilidad (MOV).

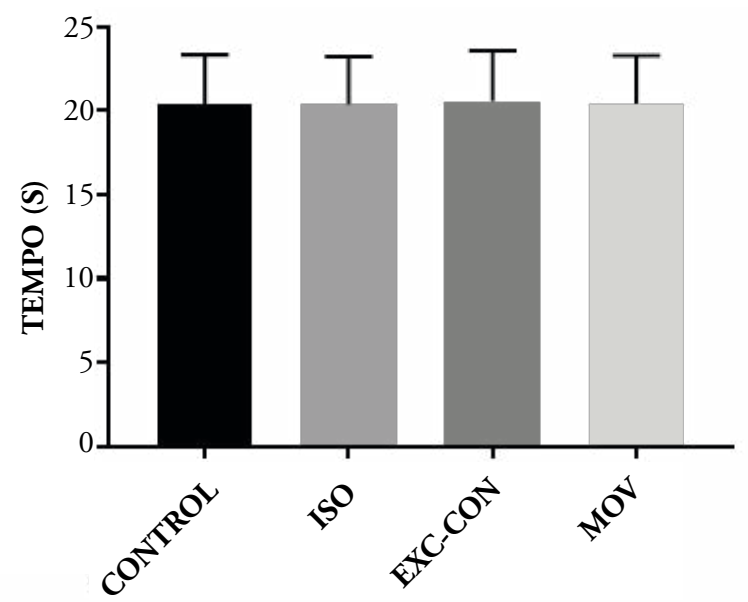

FIGURA 2 - Prueba de velocidad en Natación de 25 metros libres en grupos: control (CON), isometría (ISO), excéntrico - concéntrico (EX - CON) y movilidad (MOV).

\section{Discusión}

La activación se ha abierto camino entre las nuevas corrientes de entrenamiento y rendimiento en los últimos años, de eso no hay duda. Variados estudios avalan la mejora de los deportistas con la aplicación de una correcta técnica de potenciación post activación ${ }^{12}$. RADCLIFFE y RADCLIFFE ${ }^{13}$ encontraron mejoras en 
el rendimiento de salto después de un protocolo de activación con cargas máximas. Algunos estudios utilizaron cargas determinadas a partir de evaluaciones previas, otros utilizaron la repetición máxima $(\mathrm{RM})$ para buscar una óptima aplicación de PAP. En este estudio se buscó determinar los efectos de una activación sin carga en el rendimiento en sprint de 25 metros estilo libre con salida baja en natación, esto considerando que los sujetos no eran deportistas de alto rendimiento, aun así, los resultados muestran que las activaciones sin carga no produjeron efectos en el rendimiento deportivo (tiempo obtenido), es importante considerar, que a pesar de no haber mejoras en el tiempo, se observó un aumento de la fuerza ejercida en el agua cuando los sujetos fueron sometidos al protocolo de isometría (ISO), esto evidencia que un aumento de la fuerza no necesariamente produce una mejora en el rendimiento en sprint de natación de 25 metros estilo libre, lo que muestra que la activación con isometría produjo un aumento en la fuerza pero esto no se tradujo en una reducción del tiempo durante la prueba. Un estudio realizado en atletas de elite evidenció que ejercicios pliométricos no producen mejoras en el rendimiento en pruebas de velocidad ${ }^{14}$. En cuanto a las intervenciones en base a ejercicios con carga, una investigación realizada por BAKER ${ }^{15}$ en la cual intervino a rugbistas profesionales con ejercicios de potencia de tren superior en banco plano aplicando seis repeticiones al 65\% de una repetición máxima (desde ahora $1 \mathrm{RM}$ ), evidenció mejoras en la potencia de salida. CHIu et al. ${ }^{16}$ aplicaron un trabajo de miembro inferior para potenciación post activación en sujetos entrenados de nivel recreativo en base al protocolo de una repetición al 90\% de 1RM, encontrándose efectos positivos en la potencia después de la intervención. Otro aspecto a considerar, tal y como menciona $\mathrm{SALE}^{17}$, es que la coordinación del estímulo con la fatiga ha de ser el óptimo para de esta manera no pasar por alto el trabajo previo. BAKER ${ }^{15}$ señala que habría que estudiar qué sucede si se trabaja con cargas altas en ejercicios de tren superior con un carácter de esfuerzo bajo, para no fatigar en exceso a los músculos. Sin embargo diversos estudios hacen referencia a que esta "coordinación" tiene una estricta relación con el tiempo de pausa entre la aplicación de la carga y el estímulo que se busca, en este sentido, una revisión sistemática realizada por DeRENNE ${ }^{18}$ encontró que los protocolos utilizados para la recuperación posterior a la aplicación a la PAP flúctan entre los 3 y 12 minutos con un promedio de 5 minutos. Claramente esto se determinará dependiendo del deporte en que se aplica, como por ejemplo en un deporte con mayor explosividad, como atletismo en velocidad, saltos, velocidad en natación y salidas en esta misma. En el atletismo hay un mayor impacto y carga en sus trabajos, por ende puede que sea más apropiado utilizar PAP con carga, y esto quizás trae consigo una mayor pausa para poder de esta manera "coordinar" PAP con su estímulo final y no caer antes en la fatiga ${ }^{18}$. Considerando de esta forma la natación un deporte que demande menor impacto que el atletismo, sea más considerable utilizar otros estímulos, como el peso corporal del individuo ${ }^{19}$. En un estudio realizado en nadadores de 50 metros, se utilizó pull up o "dominada" para estimular el tren superior de los nadadores, no registrándose diferencias significativas en el rendimiento ${ }^{20}$. La mayoría de las veces los estudios utilizan carga basada en porcentajes de la repetición máxima (1RM) como estímulo previo, LINDER et al. ${ }^{21}$ lo utilizaron con atletas femeninas que se desempeñaban en 100 metros lisos, aplicando una serie previa de 4 repeticiones máximas de sentadilla, en aquel estudio se utilizaron movimientos dinámicos, encontrando efectos positivos en la velocidad cuando se aplicó la PAP, en este caso es preciso considerar que la mejoría en el rendimiento muscular después de la entrada en calor con sobrecarga depende del equilibrio entre la fatiga muscular y la potenciación muscular. Otro estudio realizado en mujeres mostró una mejora del 3,3\% en el salto vertical, luego de aplicar una serie de sentadillas isométricas ${ }^{22}$. Un estudio donde investigaron los efectos que generaba la PAP durante la fase de salida y los primeros 15 metros en nadadores de velocidad, arrojó diferencias significativas en todos los indicadores de fuerza, pero no así en el rendimiento obtenido ${ }^{19}$. Esto confirma lo expuesto anteriormente en relación a que la PAP tendría mejores resultados orientada a deportes basados en movimientos explosivos y únicos fuera del medio acuático. Cabe destacar y mencionar que, al momento de hablar de una distancia final en natación, atletismo o alguna otra ejecución explosiva, esto puede ser determinante e inclusive un factor final de victoria. Con esto último, se debe considerar que también depende del nivel de entrenamiento que tenga el sujeto de estudio, así específicamente los deportistas más fuertes y mejor entrenados pueden obtener mayores beneficios de la $\mathrm{PAP}^{23}$. Por lo tanto es más probable es que si se aplican cargas altas con sujetos no entrenados estos puedan llegar al punto de fatiga mucho antes que si se aplicará otro método, como el isométrico. Por esto es interesante determinar los efectos de activaciones sin carga o con cargas bajas en sujetos poco entrenados, esto porque los mecanismos distintos del aumento de la temperatura muscular y la activación pueden ser importantes para la mejora de PAP a corto plazo ${ }^{24}$. También se debe considerar que la temporalidad de la 
PAP cambia en gran proporción entre los individuos, la variabilidad que puede experimentar la PAP según cada sujeto debe ser considerada a la hora de esperar resultados después de aplicar un protocolo de PAP en deportistas ${ }^{22}$. En síntesis se debe considerar antes de poder aplicar un método de PAP el deporte que practica el sujeto de estudio y el estado de entrenamiento del sujeto, para de esta manera determinar el tipo de PAP que se aplicará. La potenciación se puede visualizar como un fenómeno fisiológico desencadenado a través de diferentes modalidades o estrategias de activación, el cual tendrá una denominación diferente en función del método utilizado para provocarla ${ }^{17}$. En cuanto al rendimiento deportivo, este se puede ver influenciado luego de una contracción voluntaria máxima, lo que ocurre cuando se realiza activación a través de contracciones isométricas máximas, esto produce en sujetos entrenados un efecto positivo en el rendimiento deportivo $^{25}$, debido a esto es preciso considerar que en la natación el rendimiento depende de la relación entre la fuerza aplicada y la técnica ejecutada, por esto, no necesariamente un aumento de la fuerza va a desencadenar un aumento del rendimiento, lo que se pudo apreciar en esta investigación. $\mathrm{Al}$ respecto, un estudio realizado en ciclistas de nivel internacional evidenció que la potenciación con ejercicios isométricos produjo un aumento en la cadencia y potencia promedio $^{26}$, el mismo efecto positivo produjo la activación con ejercicios isométricos en la altura de salto y potencia de salida en pesistas ${ }^{27}$, lo que justificaría el aumento de la fuerza y los resultados obtenidos en el presente estudio. Se concluye que los protocolos utilizados en el estudio no produjeron un efecto positivo en el rendimiento deportivo en nadadores de nivel recreativo. En base a esto, se hace necesario y se sugiere seguir probando distintos métodos de PAP en natación que vayan de la mano con la mejora y optimización de la técnica de nado.

\section{Abstract \\ Activation protocols and their effect on sports performance in recreational swimmers in Vina del Mar}

The aim of this study was to determine the effects of three protocols muscle activation upon force and time in a test of 25-meter freestyle with low output in a group of swimmers of recreational level from Vina del Mar, Chile. Study participants were 31 subjects with an average age of $21.86 \pm 2.38$ years. These were divided into four participation groups based on a cross method random, the groups were divided into: control group (CON), group of isometric exercise (ISO), group of eccentric and concentric exercise (EX - CON) and group joint mobility (MA). A training load equivalent to the body weight of the subject was used in all protocols. The variables evaluated: time in seconds in 25-meter freestyle with low output and maximum force recorded through the strength model monitor FMON-1 of the brand ART-OFICIO ${ }^{\circledR}$. The results showed that activation with isometrics (ISO) had positive response $(p=0.020)$ in the variable force $(N)$. Warm-up protocols did not produce decrease in time for the test of 25 meters $(p>0.05)$. These results lead to the conclusion that the isometry increased strength in the water, but this increase in strength did not reflect a decrease in time obtained in sprint swimming, other protocols did not produce variation in time or in force, It is suggested further research of the benefits of a prior activation to a sports effort, and in particular to investigate the benefits of different post activation potentiation protocols and tests prior intervention sprint swimming

KEYWORDS: Swimming; Activation; Performance; Strength.

\section{Bibliografía}

1. McLaren T, King DL, Sforzo GA. Sustainability and repeatability of postactivation potentiation. J Sports Med Phys Fitness. 2017;57(7-8):930-5.

2. Ojeda ÁH, Ríos LC, Barrilao RG, Serrano PC. Acute effect of a complex training protocol of back squats on 30-m sprint times of elite male military athletes. J Phys Ther Sci. 2016;28(3):752-6.

3. Gago P, Arndt A, Tarassova O, Ekblom MM. Post activation potentiation can be induced without impairing tendon stiffness. Eur J Appl Physiol. 2014;114(11):2299-308. 
4. Wood LK, Brooks SV. Ten weeks of treadmill running decreases stiffness and increases collagen turnover in tendons of old mice. J Orthop Res. 2016;34(2):346-53.

5. Barbosa AC, Barroso R, Andries Jr O. Post-activation potentiation in propulsive force after specific swimming strength training. Int J Sports Med. 2016;37(4):313-7.

6. Rassier D. The effects of length on fatigue and twitch potentiation in human skeletal muscle. Clin Physiol. 2000;20(6):474-82.

7. Verkhoshansky Y, Siff MC. Superentrenamiento. Barcelona: Paidotribo; 2000.

8. Zatsiorsky VM, Kraemer WJ. Science and practice of strength training. Champaign: Human Kinetics; 1995.

9. Platonov VN. El entrenamiento deportivo: teoría y metodología. Barcelona: Paidotribo; 1995.

10. Abbate F, Sargeant A, Verdijk PW, de Haan A. Effects of high-frequency initial pulses and posttetanic potentiation on power output of skeletal muscle. J Appl Physiol. 2000;88(1):35-40

11. Rushall BS. Swimming energy training in the $21^{\text {st }}$ century: the justification for radical changes. Swimming Sci Bull. 2011;39:1-59.

12. Creekmur CC, Haworth JL, Cox RH, Walsh MS. Effects of plyometrics performed during warm-up on 20 and 40 meter sprint performance. J Sports Med Phys Fitness. 2017;57(5):550-5

13. Radcliffe J, Radcliffe J. Effects of different warm-up protocols on peak power output during a single response jump task. Med Sci Sports Exerc. 1996;28(5):189.

14. Kümmel J, Bergmann J, Prieske O, Kramer A, Granacher U, Gruber M. Effects of conditioning hops on drop jump and sprint performance: a randomized crossover pilot study in elite athletes. BMC Sports Sci Med Rehabil. 2016:8:1.

15. Baker D. Acute effect of alternating heavy and light resistances on power output during upper-body complex power training. J Strength Cond Res. 2003;17(3):493-7.

16. Chiu LZ, Fry AC, Weiss LW, Schilling BK, Brown LE, Smith SL. Postactivation potentiation responses in athletic and recreationally trained individuals. J Strength Cond Res. 2003;17(4):671-7.

17. Sale DG. Postactivation potentiation: role in human performance. Exerc Sport Sci Rev. 2002;30(3):138-43.

18. DeRenne C. Effects of postactivation potentiation warm-up in male and female sport performances: a brief review. Strength Cond J. 2010;32(6):58-64

19. Kilduff LP, Cunningham DJ, Owen NJ, West DJ, Bracken RM, Cook CJ. Effect of postactivation potentiation on swimming starts in international sprint swimmers. J Strength Cond Res. 2011;25(9):2418-23.

20. Sarramian VG, Turner AN, Greenhalgh AK. Effect of postactivation potentiation on fifty-meter freestyle in national swimmers. J Strength Cond Res. 2015;29(4):1003-9.

21. Linder EE, Prins JH, Murata NM, DeRenne C, Morgan CF, Solomon JR. Effects of preload 4 repetition maximum on 100-m sprint times in collegiate women. J Strength Cond Res. 2010;24(5):1184-90.

22. Guellich A, Schmidtbleicher D. MVC-induced short-term potentiation of explosive force. N Stud Athl. 1996;11(4):67-81.

23. Duthie GM, Young WB, Aitken DA. The acute effects of heavy loads on jump squat performance: an evaluation of the complex and contrast methods of power development. J Strength Cond Res. 2002;16(4):530-8.

24. Barnes MJ, Petterson A, Cochrane DJ. Effects of different warm-up modalities on power output during the high pull. J Sports Sci. 2017;35(10):976-81.

25. Cloak R, Lane A, Wyon M. Professional soccer player neuromuscular responses and perceptions to acute whole body vibration differ from amateur counterparts. J Sports Sci Med. 2016;15(1):57-64.

26. Munro LA, Stannard SR, Fink PW, Foskett A. Potentiation of sprint cycling performance: the effects of a high-inertia ergometer warm-up. J Sports Sci. 2017;35(14):1442-50.

27. Rixon KP, Lamont HS, Bemben MG. Influence of type of muscle contraction, gender, and lifting experience on postactivation potentiation performance. J Strength Cond Res. 2007;21(2):500-5.

\author{
DIRECCIÓN: \\ Rodrigo Yañez \\ El Bosque, 1290 \\ 2340000 - Valparaiso - CHILE \\ e-mail: medicodeportivochile@gmail.com \\ Presentado para publicación: 06/11/2016 \\ $1^{\text {a }}$ Revisión: 23/05/2017 \\ $2^{\text {a }}$ Revisión: 19/08/2017 \\ Aprobado: 18/09/2017
}

Rev Bras Educ Fís Esporte, (São Paulo) 2019 Jan-Mar;33(1):21-7 • 27 